\title{
Patient Preferences for Test Result Notification
}

\author{
Samuel K. Shultz, B.S. ${ }^{7}$, Robert Wu, M.D. M.S. ${ }^{2,3}$, John J. Matelski, M.S. ${ }^{3}$, Xin Lu, M.S. ${ }^{1,4}$, \\ and Peter Cram, M.D. M.B.A. ${ }^{7,2,3}$
}

${ }^{1}$ Carver College of Medicine, University of lowa, lowa City, IA, USA; ${ }^{2}$ Faculty of Medicine, University of Toronto, Toronto, ON, Canada; ${ }^{3}$ Division of General Internal Medicine and Geriatrics, Mt. Sinai/UHN Hospitals, Toronto, ON, Canada; ${ }^{4}$ CADRE, lowa City VA Medical Center, lowa City, IA, USA.

\begin{abstract}
IMPORTANCE: Patients are increasingly being given access to their test results, but little is known about how preferences vary with the test under consideration or the results of the test (normal or abnormal).
\end{abstract}

OBJECTIVE: This study was conducted to examine preferences for test result communication.

DESIGN, SETTING, AND PARTICIPANTS: We surveyed adults to explore their preferences for test result notification for three common diagnostic tests of varying "emotional impact” (dual-energy x-ray absorptiometry [DXA], genital herpes, and cancer biopsy) when test results were 1) normal and 2) abnormal. We conducted our survey between June and August 2012 on the campus of an academic medical center. For each scenario, subjects were asked to rank seven methods that might be used to communicate test results (letter, unsecured email, secured email, text message, telephone call, secure Web portal, office visit) in order of acceptability.

MAIN OUTCOME MEASURES: The main measures were the percentage of respondents who ranked a particular test result notification method favorably and the percentage who ranked it as unacceptable.

RESULTS: When test results were normal, subjects' notification preferences were generally similar for DXA, herpes and cancer biopsy, with telephone and letter ranked most favorably for all three tests. Conversely, text message and unsecured email were viewed as unacceptable notification methods for normal results by $45.0-55.0 \%$ of subjects across all three tests. When test results were abnormal, office visits became more popular. A higher proportion of subjects ranked office visits as their most preferred notification method for our test with high "emotional impact" (cancer biopsy) (38.4\%) as compared to DXA $(28.2 \%)$ and herpes $(27.9 \%)(P=0.02)$. For most test scenarios, younger subjects appeared to rank electronic communication modalities (secure email or Web portal) higher than older subjects, though this difference did not reach statistical significance $(P=0.29)$.

CONCLUSIONS: Preferences for test result notification can differ substantially depending upon the test under consideration and results of the test. Providers and health care systems should consider these factors when deciding how to communicate results to patients.

Electronic supplementary material The online version of this article (doi:10.1007/s11606-015-3344-0) contains supplementary material, which is available to authorized users.

Received May 21, 2014

Revised November 5, 2014

Accepted April 1, 2015

Published online May 6, 2015
KEY WORDS: test results notification; patient preferences.

J Gen Intern Med 30(11):1651-6

DOI: $10.1007 / \mathrm{s} 11606-015-3344-0$

๑) Society of General Internal Medicine 2015

\section{INTRODUCTION}

In order to provide high-quality patient care, all test results should be tracked, reviewed, and acted upon. ${ }^{1}$ However, there is now abundant evidence that test result management practices are highly variable, ${ }^{2,3}$ and in the most serious cases, missed test results can lead to real patient harm. ${ }^{4-6}$

Studies have demonstrated that many providers lack systems for ensuring that the tests they order are completed and for reliably communicating the results to patients. ${ }^{2,7,8} \mathrm{Re}$ search has also demonstrated that both normal and abnormal test results are commonly missed, even in health care systems with advanced electronic health records (EHRs), ${ }^{8,9}$ and that providers miss $1-10 \%$ of abnormal test results. ${ }^{9,10}$ A systematic review by Callen et al. found that, across 19 published studies, $6.8-62 \%$ of lab tests were not followed up on. ${ }^{11}$ It would not be an overstatement to conclude that the health care system lacks mechanisms for ensuring that test results are consistently conveyed to patients. ${ }^{3,12}$

As appreciation of problems inherent in test result reporting processes has increased, a number of potential solutions have been explored. One strategy has involved the development of advanced computerized in-boxes that assist providers in reviewing results, initiating actions (e.g., referrals, office visits) for abnormal results, and notifying patients. ${ }^{13,14}$ Another potential solution has entailed creating processes whereby testing centers routinely provide test results directly to patients; such systems range from sending test results by mail to sophisticated Web-based electronic patient portals. ${ }^{15}$ While there is growing momentum in favor of sharing test results with patients, few studies have empirically evaluated patients' preferences for receiving notification of their test results. ${ }^{16-18}$ Moreover, we are unaware of any studies that have evaluated how preferences vary with patient demographics, the emotional impact of the particular test being considered, and whether the test result was normal or abnormal.

We conducted a survey to examine how adults would want to be informed of three different test results of varying emotional impact (dual-energy x-ray absorptiometry [DXA] scan, genital herpes, and cancer) and how notification preferences 
would vary depending upon whether the test result was normal or abnormal.

\section{METHODS}

\section{Study Setting}

Our study was conducted on the campus of a major academic medical center located in the U.S. Midwest. The hospital, including all inpatient and outpatient clinics, uses the Epic EHR (Epic Systems Corporation, Verona, WI, USA). Patients have the ability to register for access to Epic's MyChart patient portal that allows patients the option of communicating with their health care team through a secure Web-based application. MyChart also allows patients to review and access their own test results. At the time of our study, approximately $28 \%$ of patients had signed up for MyChart (personal communication, Dr. Prakash Nadkarni).

\section{Survey Development}

We developed a survey to examine patients' preferences for notification of their test results. Our work built upon prior research that we and others have performed in this area. ${ }^{10,16,17,19}$ We collected data on survey respondents' demographic (age, race, sex), educational attainment (categorized as high school or less, some college, completed college, and graduate school or above), and baseline health status (categorized as excellent, very good, good, fair, or poor) information. The full survey instrument is included as Appendix 1.

In keeping with our study objective, we identified three tests of varying "emotional impact," following a design we had used previously. ${ }^{10}$ We selected one test of low emotional impact (DXA scan), one test of intermediate impact (genital herpes), and one of high impact (biopsy for possible cancer [breast for women, prostate for men]).

We developed a brief scenario for each of the three tests (DXA, herpes, cancer) coupled with each potential result (test normal and test abnormal), for a total of six scenarios administered to each subject (see Appendix 1). For each scenario, subjects were given a list of seven ways that they could be notified of their results (mailed letter, unsecured email, secure email, text message, telephone call, secure internet Web portal, and office visit); there was an additional option to select "other," with space for free-text entry. Secure email was described as email with additional security above and beyond that of usual email systems (i.e., Gmail, Yahoo). Each subject was instructed to rank the potential methods for result notification from most preferred to least preferred; subjects were also instructed to mark any methods that they found unacceptable, and these tests were eliminated from the ranking exercise. Thus, each subject completed the ranking exercise for six different test result scenarios (DXA normal, DXA abnormal, herpes normal, herpes abnormal, cancer biopsy normal, and cancer biopsy abnormal).

\section{Survey Administration}

We initially developed a paper-based draft of our survey, and then converted the survey instrument into an electronic version (Qualtrics LLC, Provo, UT, USA) that was administered using a tablet computer. We conducted pilot testing to ensure that the material was clearly worded and that our test selections were logical. After updating each scenario, we administered the survey to a random sample of subjects drawn from five different clinic waiting rooms within a large tertiary care academic medical center as well as from several public locations visited by faculty and students on our medical campus. We did not target patients over family members, and likewise did not make efforts to include or exclude students or staff. A member of our study team, SS, would enter one of the recruitment locations and randomly approach the nearest potential participant who appeared at or above our minimum age requirement of 18 years; if an individual refused to participate, we attempted to obtain basic demographic data, including age, race, and sex, to allow for comparison of patients who did and did not agree to participate.

Participants who agreed to participate were invited to complete the survey, either where they were, or they could choose to be escorted to a private office for greater privacy. Each respondent was given the tablet computer; assistance with both the technology and content of the survey was provided by one of the study team members (SS) on an as-needed basis (Appendix 1).

\section{Statistical Analyses}

First, we compared the demographic characteristics of subjects who did and did not agree to participate in our study using relevant methods ( $t$ test, chi-square statistic). We then examined the sociodemographic characteristics of subjects who completed the survey, including age, sex, race, and educational attainment. Continuous variables were summarized as mean and standard deviation (SD), while categorical variables were described using proportions (percentages).

Second, we examined subjects' notification preferences for each of the three tests (DXA, herpes, and cancer biopsy) when the results were normal and when the results were abnormal (six scenarios in total). More specifically, for each scenario we examined the percentage of subjects who ranked a given result notification method (e.g., office visit, telephone call) as their top choice (each subject was allowed a single top choice for each scenario). We also calculated the percentage of respondents who ranked a particular method as unacceptable for each of the six scenarios (subjects could rank multiple methods as unacceptable for each scenario).

After calculating the rankings, for each of the six scenarios, we compared the percentage of subjects who designated a 
given notification method (e.g., mailed letter) as their "top choice" when the test was normal (e.g., normal DXA) versus abnormal (e.g., abnormal DXA) using the Wald chi-square test; this comparison allowed us to ascertain whether top choices varied depending upon whether results were normal or abnormal. We conducted similar analyses to evaluate notification methods that were deemed unacceptable; in particular, we compared the percentage of subjects who designated a given notification method as unacceptable when a given test was normal (e.g., herpes normal) versus abnormal (e.g., herpes abnormal). To ensure the robustness of our findings, we also evaluated preferred notification by focusing on whether a given method was ranked amongst a subject's "top 3" choices as another measure of examining which reporting methods were viewed favorably.

Third, we examined whether subjects' preferences for electronic notification (secured email or Web portal) varied with age. Specifically, we stratified subjects into three age categories: $18-30$ years, $31-60$ years, and 61 years and above. We then compared the percentage of subjects in each age stratum who ranked secure electronic notification methods as their top choice. This study was approved by the University of Iowa institutional review board. All analyses were conducted using $\mathrm{R}$ version 3.0.2.

\section{RESULTS}

We approached 431 subjects, of whom 305 (70.8\%) completed the survey. Subjects who agreed and did not agree to participate in our study were similar with respect to age and race, though refusal rates were higher for men than women $(P<0.01)$. The mean age of study participants was 47.7 years, and $33.4 \%$ were men (Table 1). Our population was well educated, with more than $50.0 \%$ having completed college; $60.6 \%$ rated their health status as very good or excellent (Table 1).

Table 1 Characteristics of Study Subjects

\begin{tabular}{ll}
\hline Characteristic & N=305 \\
\hline Demographics & \\
Age, mean (sd) & $47.7(18.4)$ \\
Male, number (\%) & $102(33.4)$ \\
Race, number (\%) & \\
White & $270(89.1)$ \\
Black & $6(2.0)$ \\
Hispanic & $5(1.7)$ \\
Asian & $11(3.6)$ \\
Native American & $1(0.3)$ \\
Other & $3(1.0)$ \\
Multiracial & $7(2.3)$ \\
Education & $69(22.6)$ \\
High school or less, number (\%) & $76(24.9)$ \\
Some college, number (\%) & $64(21.0)$ \\
Completed college, number (\%) & $96(31.5)$ \\
Graduate school, number (\%) & \\
Health status & $62(20.3)$ \\
Excellent, number (\%) & $123(40.3)$ \\
Very good, number (\%) & $85(27.9)$ \\
Good, number (\%) & $35(11.5)$ \\
Fair or poor, number (\%) &
\end{tabular}

*2 subjects did not report race
For notification of normal test results, subjects' preferences were generally clinically similar across the different test scenarios (Table 2, Columns 1 and 3). For example, a telephone call was selected as the most preferred method for notification for normal results for all three tests (telephone call ranked as top choice by $33.8 \%$ for DXA, $40.3 \%$ for herpes, $43.0 \%$ for cancer; $P=0.12$ for difference across tests). Likewise, unsecured email was viewed as unacceptable when results were normal for DXA, herpes, and cancer by 48.2, 52.1 and $54.8 \%$ of subjects, respectively $(P=0.19)$ (Table 2 and eFigure 1)

For notification of abnormal test results, the preferred methods of result notification differed somewhat across tests (Table 2, Columns 2 and 4). For example, a significantly higher proportion of patients ranked an office visit as their top choice for an abnormal cancer test (38.4\%) as compared to DXA $(28.2 \%)$ and herpes $(27.9 \%)(P=0.002)$. Alternatively, with regard to notification methods viewed as unacceptable for abnormal tests (Table 2, Column 4), the percentage of subjects who viewed text messaging as unacceptable was similar for cancer $(58.7 \%)$ and herpes $(57.1 \%)$ as compared to DXA $(53.4 \%)(P=0.20)$, suggesting no significant differences.

In viewing results from a different perspective, respondent preferences for result notification were substantially different when comparing preferences for normal versus abnormal results within each of the three diagnostic tests (e.g., DXA normal versus DXA abnormal) (Table 2 and eFigure 2). For example, $33.8 \%$ of subjects ranked telephone as their top choice for a normal DXA (Table 2, Column 1) as compared to $43.3 \%$ (Table 2, Column 2) for abnormal DXA ( $P=0.02)$. Likewise, a mailed letter was viewed as unacceptable by $11.2 \%$ of subjects when DXA was normal but $22.3 \%$ when DXA was abnormal $(P<0.001)$. Trends were similar in comparisons of preferences for normal versus abnormal results for herpes and cancer.

In supplementary analyses, we found that acceptance of electronic notification, defined as secure email or Web portal, appeared to be higher for younger subjects than older subjects, though differences were not statistically significant (Fig. 1). For example, for a normal herpes test, $21.6 \%$ of subjects aged 18-30 years ranked an electronic source as their top choice for communication, compared to $28.8 \%$ of subjects aged 61 and over $(P=0.29)$.

\section{DISCUSSION}

A confluence of societal, regulatory and technological factors are driving the health care system to improve patients' access to their test results. However, there is little empirical data describing how patients want to receive their test results and how preferences might change from test to test and between normal and abnormal results. In a study of more than 300 subjects, we note a number of important findings. First, we found that telephone calls and office visits were generally viewed most favorably, while unsecured electronic communication (unsecured email, text messaging) was viewed as 
Table 2 Preferences for Receiving Results $(N=305)$

\begin{tabular}{|c|c|c|c|c|c|c|}
\hline & $\begin{array}{l}\text { Percentage of } \\
\text { subjects ranking } \\
\text { notification method } \\
\text { as top choice when } \\
\text { results are normal } \\
\text { (1) }\end{array}$ & $\begin{array}{l}\text { Percentage of } \\
\text { subjects ranking } \\
\text { notification method } \\
\text { as top choice when } \\
\text { results are } \\
\text { abnormal (2) }\end{array}$ & $\begin{array}{l}\text { Percentage of } \\
\text { subjects ranking } \\
\text { notification method } \\
\text { as unacceptable } \\
\text { when results are } \\
\text { normal (3) }\end{array}$ & $\begin{array}{l}\text { Percentage of } \\
\text { subjects ranking } \\
\text { notification method } \\
\text { as unacceptable } \\
\text { when results are } \\
\text { abnormal (4) }\end{array}$ & $\begin{array}{l}P \text { values } \\
\text { for } 1 \text { vs } 2\end{array}$ & $\begin{array}{l}P \text { values } \\
\text { for } 3 \text { vs } 4\end{array}$ \\
\hline \multicolumn{7}{|l|}{ DXA, } \\
\hline \multicolumn{7}{|l|}{ Number, $(\%)$} \\
\hline Letter by U.S. Mail & $64(21.0)$ & $29(9.5)$ & $34(11.2)$ & $68(22.3)$ & 0.04 & $<<0.01$ \\
\hline Unsecured email & $8(2.6)$ & $6(2.0)$ & $147(48.2)$ & $163(53.4)$ & 0.60 & 0.20 \\
\hline Secure email & $47(15.4)$ & $26(8.5)$ & $32(10.5)$ & $50(16.4)$ & $<0.01$ & 0.03 \\
\hline Text message & $10(3.3)$ & $5(1.6)$ & $139(45.6)$ & $163(53.4)$ & 0.64 & 0.05 \\
\hline Telephone call & $103(33.8)$ & $132(43.3)$ & $16(5.3)$ & $15(4.9)$ & 0.02 & 0.85 \\
\hline Secure Web portal & $48(15.7)$ & $19(6.2)$ & $54(17.7)$ & $62(20.3)$ & $<<0.01$ & 0.41 \\
\hline Office visit & $23(7.5)$ & $86(28.2)$ & $50(16.4)$ & $22(7.2)$ & $<<0.01$ & $<0.01$ \\
\hline \multicolumn{7}{|l|}{ Herpes, } \\
\hline \multicolumn{7}{|l|}{ Number, $(\%)$} \\
\hline Letter by U.S. Mail & $55(18.0)$ & $34(11.2)$ & $46(15.1)$ & $90(29.5)$ & 0.93 & $<<0.01$ \\
\hline Unsecured email & $5(1.6)$ & $4(1.3)$ & $159(52.1)$ & $177(58.0)$ & 0.22 & 0.14 \\
\hline Secure email & $46(15.1)$ & $23(7.5)$ & $41(13.4)$ & $58(19.0)$ & $<<0.01$ & 0.06 \\
\hline Text message & $8(2.6)$ & $5(1.6)$ & $157(51.5)$ & $174(57.1)$ & 0.78 & 0.17 \\
\hline Telephone call & $123(40.3)$ & $125(41.0)$ & $13(4.3)$ & $22(7.2)$ & 0.81 & 0.12 \\
\hline Secure Web portal & $42(13.8)$ & $27(8.9)$ & $51(16.7)$ & $64(21.0)$ & 0.36 & 0.18 \\
\hline Office visit & $24(7.9)$ & $85(27.9)$ & $43(14.1)$ & $25(8.2)$ & $<<0.01$ & 0.02 \\
\hline \multicolumn{7}{|l|}{ Cancer, } \\
\hline \multicolumn{7}{|l|}{ Number, $(\%)$} \\
\hline Letter by U.S. Mail & $56(18.4)$ & $18(5.9)$ & $51(16.7)$ & $104(34.1)$ & 0.05 & $<<0.01$ \\
\hline Unsecured email & $6(2.0)$ & $4(1.3)$ & $167(54.8)$ & $182(59.7)$ & 0.15 & 0.22 \\
\hline Secure email & $33(10.8)$ & $17(5.6)$ & $47(15.4)$ & $79(25.9)$ & 0.02 & $<0.01$ \\
\hline Text message & $8(2.6)$ & $2(0.7)$ & 149 (48.9) & $179(58.7)$ & 0.64 & 0.02 \\
\hline Telephone call & $131(43.0)$ & $132(43.3)$ & $8(2.6)$ & $26(8.5)$ & 0.57 & $<0.01$ \\
\hline Secure Web portal & $36(11.8)$ & $14(4.6)$ & $54(17.7)$ & $84(27.5)$ & 0.01 & $<0.01$ \\
\hline Office visit & $33(10.8)$ & $117(38.4)$ & $36(11.8)$ & $18(5.9)$ & $<<0.01$ & 0.01 \\
\hline
\end{tabular}

undesirable. Second, we found that preferred methods of notification changed relatively little from test to test, but varied substantially when results switched from normal to abnormal. Third, we found that acceptance of electronic communication was higher among younger subjects than older ones. In total, our results hint at the complexity of communicating test results to patients.

A number of our findings merit discussion. First, it is important to understand the rationale for improving the communication of test results to patients. There is now robust evidence that test results are often missed and that providers lack reliable systems for communicating results to patients. ${ }^{3,8,20-25}$ In one cross-sectional study, only $41 \%$ of discharged patients had test results returned to them, and only $61 \%$ of caregivers were aware of the results. ${ }^{26}$ Most primary care physicians see hundreds of tests per week, and volume alone is a challenge. ${ }^{12}$ Implementing systems whereby test results are routinely "pushed out" to patients has been proposed as one way to ensure that important results are not missed. Ignoring the patient safety benefits that might accrue from routinely conveying test results to patients, patients consistently report that they want to know their test results whether normal or abnormal. ${ }^{17,19}$ For example, Baldwin and colleagues found that $90 \%$ of patients wanted to be notified of all test results, ${ }^{16}$ and a recent trial by Delbanco and colleagues found that providing open access to medical records was well received by patients and providers alike. ${ }^{27}$
Several EHR developers have created patient portals, such as Epic's MyChart or the Veterans Health Administration's My HealtheVet, to meliorate communication with patients. ${ }^{28,29}$ Both systems have functions that allow patients to review their own test results; more detailed nuances including the types of tests available for review, when results are posted (e.g., immediately upon completion of testing or only after provider review and approval) can often by controlled by individual providers or computer system administrators. Little attention has been given to whether such portals are viewed favorably by patients or to precisely which tests or results might not be suitable for communication via portals.

Second, we found that subjects generally preferred telephone calls and office visits, while viewing others communication routes less favorably - in particular, text messaging and unsecured email. This finding is important, and it has practical value. The greater security afforded by Web portals and encrypted email systems comes at a cost of increased hassle for patients and providers. We believe that there is an inherent conflict between "ease of access" and "data security," suggesting that a perfect solution will be difficult to come by. Our findings suggest that health systems and EHR vendors can take concrete steps to facilitate the types of communication that subjects prefer, while discouraging the use of unsecured email and text messages in most situations.

Third, our results demonstrated surprising consistency in terms of the notification methods that were viewed favorably 


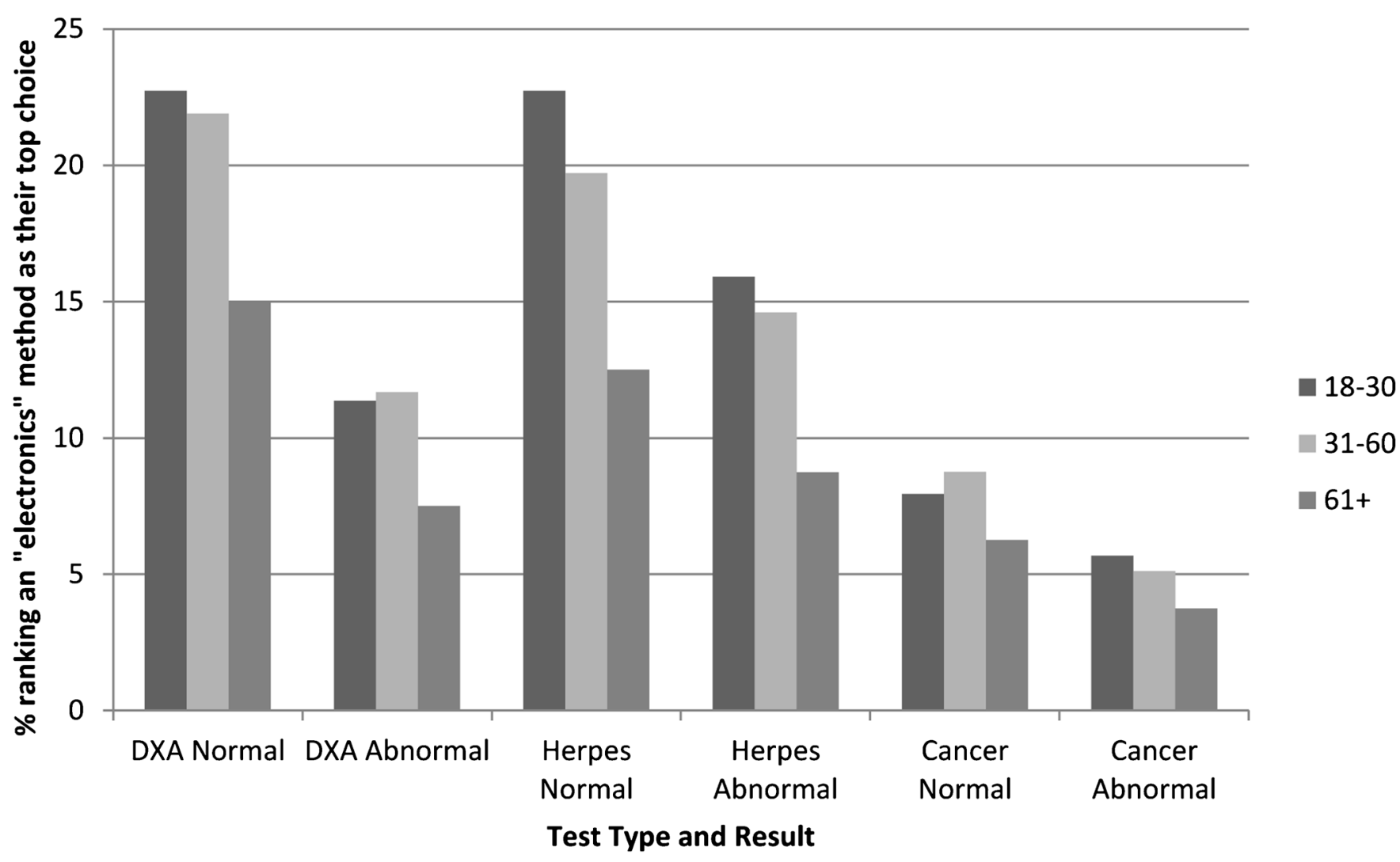

Figure 1 Relationship between electronic (secure email or Web portal) methods of notification, age, and test scenario. "Electronic method" was defined as notification by secure email or Web portal. For most test scenarios, younger subjects (18-30) appeared more likely to rank electronic methods highly than older subjects (age 61+), though this difference did not reach statistical significance (Wald chi-square P values: DXA normal, $P=0.66$; DXA abnormal, $P=0.69$; herpes normal, $P=0.29$; herpes abnormal, $P=0.44$; cancer normal, $P=0.96$; cancer abnormal, $P=0.32$ ).

and unfavorably as we moved from DXA to herpes to cancer. From this perspective, patient preferences seemed to differ from provider preferences for test result notification. ${ }^{10}$ At the same time, there were modest shifts in preference when we moved from normal to abnormal test results. For example, office visits were increasingly preferred when results were abnormal as opposed to normal. Alternatively, telephone calls were viewed favorably in virtually all scenarios. We also found evidence that preferences for communication differed between younger and older patients, with older patients viewing electronic notification methods less favorably. In aggregate, these results suggest that, ideally, test result reporting would be tailored to the test under consideration, the results of the test, and the age of the recipient. Of course, creating a system that could account for just these three factors is far beyond any test result reporting system we have encountered in clinical practice.

Fourth, it is important to discuss how our findings relate to the current legislative environment. The Department of Health and Human Services recently issued an important final rule amending HIPAA and the Clinical Laboratory Improvement Act (CLIA) in order to facilitate greater patient access to test results. ${ }^{30}$ Specifically, a February 2014 update to the CLIA now requires most laboratories to provide patients (or their delegates) with direct access to their test results. Precisely how laboratory operators respond to these regulations remains to be seen. At the state level, regulations are changing as well, with new requirements for communicating results to patients adding to an already confusing landscape ${ }^{31,32}$ It is both fascinating and frightening to see a lack of rigorous empirical research on patient preferences to inform these changes.

Several limitations of our study are important to mention. First, our study subjects were drawn from a single academic medical center campus and were generally white, well educated, and healthy. Our institution had already completed implementation of the Epic EHR, including Epic's MyChart patient portal. It will be important to replicate our study in other environments and among other patient populations. Second, we did not directly inquire about subjects' prior experience with various test result communication processes (e.g., Web portals) or whether subjects had registered for Epic MyChart. Third, our clinical scenarios deliberately focused upon discrete tests that would be performed infrequently for a given patient. It is unclear how preferences for communication might differ for tests that are performed routinely such as blood pressure or hemoglobin A1c. It is also important to acknowledge that, while our effort to identify tests of varying emotional impact has face validity, we are unaware of any empirical research assessing emotional impact. Fourth, we did not directly evaluate subjects' prior experiences with the tests under consideration. Fifth, all participants were given the test scenarios in the 
same order, creating the potential for order effects. Our analysis also included multiple comparisons, increasing the possibility that differences were detected in statistical testing as a consequence of mere chance (type 1 error). Lastly, an older adult may find it difficult to imagine the angst associated with genital herpes, or a younger patient might find it difficult to understand the anxiety related to osteoporosis.

Preferences for test result notification differ substantially amongst individuals based upon whether results are normal or abnormal and on the patient's age. Providers and health care systems should consider these factors when deciding how to communicate results to patients.

ACKNOWLEDGMENTS: Samuel K. Shultz, John Matelski, and Xin Lu had full access to all of the data in the study and take responsibility for the integrity of the data and the accuracy of the data analysis. Samuel K. Shultz, Xin Lu, Robert Wu, John J. Matelski, and Peter Cram are responsible for the data analysis. Peter Cram is supported by a K24 award from the National Institute of Arthritis and Musculoskeletal and Skin Diseases (NIAMS; AR062133). This work is also funded in part by R01 AG033035 from the National Institute on Aging (NIA) at the National Institute of Health (NIH). There were no other contributors and there have been no previous presentations on this research.

Conflict of Interest: The authors declare no conflicts of interest.

Corresponding Author: Samuel K. Shultz, B.S.; Carver College of Medicine, University of Iowa, 520 Clark St., Iowa City, IA 52240, USA (e-mail: samuel-shultz@uiowa.edu).

\section{REFERENCES}

1. Schiff G. Getting Results: Reliably Communicating and Acting on Critical Test Results. 1 ed. 2006: Joint Commission. 156.

2. Wahls T, Haugen T, Cram P. The continuing problem of missed test results in an integrated health system with an advanced electronic medical record. Jt Comm J Qual Patient Saf. 2007;33(8):485-492.

3. Boohaker EA, Ward RE, Uman JE, McCarthy BD. Patient notification and follow-up of abnormal test results. A physician survey. Arch. Intern. Med. 1996; 156(3):327-331.

4. Holohan TV, Colestro J, Grippi J, Converse J, Hughes M. Analysis of diagnostic error in paid malpractice claims with substandard care in a large healthcare system. South Med J. 2005;98(11):1083-1087.

5. Gandhi TK, Kachalia A, Thomas EJ, et al. Missed and delayed diagnoses in the ambulatory setting: a study of closed malpractice claims. Ann Intern Med. 2006; 145(7):488-496.

6. Poon EG, Kachalia A, Puopolo AL, Gandhi TK, Studdert DM. Cognitive errors and logistical breakdowns contributing to missed and delayed diagnoses of breast and colorectal cancers: a process analysis of closed malpractice claims. J Gen Intern Med. 2012;27(11):1416-1423.

7. Murff HJ, Gandhi TK, Karson AK, et al. Primary care physician attitudes concerning follow-up of abnormal test results and ambulatory decision support systems. Int $\mathrm{J}$ Med Inform. 2003;71(2-3):137-149.

8. Poon EG, Gandhi TK, Sequist TD, Murff HJ, Karson AS, Bates DW. "I wish I had seen this test result earlier!": dissatisfaction with test result management systems in primary care. Arch Intern Med. 2004; 164(20):2223-2228.

9. Casalino LP, Dunham D, Chin MH, et al. Frequency of failure to inform patients of clinically significant outpatient test results. Arch Intern Med. 2009; 169(12): 1123-1129.

10. Sung S, Forman-Hoffman V, Wilson MC, Cram P. Direct reporting of laboratory test results to patients by mail to enhance patient safety. J Gen Intern Med. 2006;21(10): 1075-1078.
11. Callen JL, Westbrook JI, Georgiou A, Li J. Failure to follow-up test results for ambulatory patients: a systematic review. J Gen Intern Med. 2012;27(10):1334-1348.

12. Singh H, Spitzmueller C, Petersen NJ, et al. Primary care practitioners' views on test result management in EHR-enabled health systems: a national survey. J Am Med Inform Assoc. 2013;20(4):727-735.

13. Matheny ME, Gandhi TK, Orav EJ, et al. Impact of an automated test results management system on patients' satisfaction about test result communication. Arch Intern Med. 2007;167(20):2233-2239.

14. Laxmisan A, Sittig DF, Pietz K, Espadas D, Krishnan B, Singh H. Effectiveness of an electronic health record-based intervention to improve follow-up of abnormal pathology results: a retrospective record analysis. Med Care. 2012;50(10):898-904.

15. Edmonds SW, Wolinsky FD, Christensen AJ, et al. The PAADRN study: a design for a randomized controlled practical clinical trial to improve bone health. Contemp Clin Trials. 2013;34(1):90-100.

16. Baldwin DM, Quintela J, Duclos C, Staton EW, Pace WD. Patient preferences for notification of normal laboratory test results: a report from the ASIPS Collaborative. BMC Fam Pract. 2005;6(1):11.

17. Leekha S, Thomas KG, Chaudhry R, Thomas MR. Patient preferences for and satisfaction with methods of communicating test results in a primary care practice. Jt Comm J Qual Patient Saf. 2009;35(10):497-501.

18. Grimes GC, Reis MD, Budati G, Gupta M, Forjuoh SN. Patient preferences and physician practices for laboratory test results notification. J Am Board Fam Med. 2009;22(6):670-676.

19. Cram P, Schlechte J, Rosenthal GE, Christensen AJ. Patient preference for being informed of their DXA scan results. J Clin Densitom. 2004;7(3):275-280.

20. Hysong SJ, Sawhney MK, Wilson L, et al. Understanding the management of electronic test result notifications in the outpatient setting. BMC Med Inform Decis Mak. 2011;11:22.

21. Callen J, Georgiou A, Li J, Westbrook JI. The safety implications of missed test results for hospitalised patients: a systematic review. BMJ Qual Saf. 2011;20(2):194-199.

22. Callen J, Paoloni R, Georgiou A, Prgomet M, Westbrook J. The rate of missed test results in an emergency department: an evaluation using an electronic test order and results viewing system. Methods Inf Med. 2010;49(1):37-43.

23. Wahls TL, Cram P. Proposed interventions to decrease the frequency of missed test results. Adv Health Sci Educ Theory Pract. 2009;14(Suppl 1):51-56.

24. Gordon JR, Wahls T, Carlos RC, Pipinos II, Rosenthal GE, Cram P. Failure to recognize newly identified aortic dilations in a health care system with an advanced electronic medical record. Ann Intern Med. 2009; 151(1):21-27. w5.

25. Dalal AK, Roy CL, Poon EG, et al. Impact of an automated email notification system for results of tests pending at discharge: a clusterrandomized controlled trial. J Am Med Inform Assoc. 2013.

26. Jones N. Helicobacter pylori treatment in the hospital setting: a potential model for developing quality improvement initiatives to prevent missed test results. Can J Gastroenterol. 2011;25(10):542.

27. Delbanco T, Walker J, Bell SK, et al. Inviting patients to read their doctors' notes: a quasi-experimental study and a look ahead. Ann Intern Med. 2012;157(7):461-470.

28. Serrato CA, Retecki S, Schmidt DE. MyChart-a new mode of care delivery: 2005 personal health link research report. Perm J. 2007;11(2):14-20.

29. Woods SS, Schwartz E, Tuepker A, et al. Patient experiences with full electronic access to health records and clinical notes through the My HealtheVet Personal Health Record Pilot: qualitative study. J Med Internet Res. 2013;15(3), e65.

30. Centers for Medicare and Medicaid Services. Publication of Final Rule "Clinical Laboratory Improvement Amendments (CLIA) Program and Health Insurance Portability and Accountability Act (HIPAA) Privacy Rule; Patients' Access to Test Reports. 2014 [Accessed 2015 3rd April]; Available from: http://www.cms.gov/Medicare/Provider-Enrollment-and-Certification/SurveyCertificationGenInfo/Policy-and-Memos-to-States-and-Regions-Items/Survey-and-Cert-Letter-14-11.html.

31. Dube N. Direct Patient Access to Lab Test Results. 2013 [Accessed 2015 3rd Aprill; Available from: http://www.cga.ct.gov/2013/rpt/2013-R-0151.htm.

32. Kasprak J. Patient Direct Access to Medical Test Results. 2008 [Accessed 2015 3rd April]; Available from: http://www.cga.ct.gov/2008/rpt/2008-R0419.htm. 